
\title{
28 Research Square \\ Jumpstarting nitric oxide production might help prevent early-stage breast cancer
}

Gang Ren

Xunzhen Zheng

Matthew Bommarito

Samantha Metzger

Yashna Walia

Joshua Letson

Allen Schroering

Andrea Kalinoski

David Weaver

Christopher Figy

Kam Yeung

Saori Furuta

\section{Video Abstract}

Keywords: nitric oxide, breast cancer, cancer, invasive cancer, sepiapterin, tumor, mammary gland, mouse model, cancer treatment, early-stage breast cancer, cancer prevention, nanoparticle, drug delivery, Scientific Reports, University of Toledo Health Science Campus, cancer progression, oxidative stress, TGF beta, ERBB2

Posted Date: September 23rd, 2019

DOI: https://doi.org/10.21203/rs.2.14981/v2

License: (a) (i) This work is licensed under a Creative Commons Attribution 4.0 International License. Read Full License 


\section{Abstract}

A new study shows that abnormal production of nitric oxide in the body leads to the progression of breast cancer in mice. This finding could open the door to new treatments for early-stage breast cancer that stabilize the production of this pivotal molecule. Nearly one-third of newly diagnosed breast cancers in the US are early-stage lesions. Though technically pre-cancerous, about $40 \%$ of these lesions could progress to invasive cancers. Researchers don't yet fully understand what drives these insidious formations. But various studies have reported a common link between cancer risk factors related to lifestyle-such as a high-fat diet, high alcohol consumption, and low physical activity-and abnormal production of nitric oxide. Normally, physiological stress triggers the production of large amounts of nitric oxide. This activates tissue-specific functions of neurons, muscles, immune cells, blood vessels and other specialized cells. Under unstressed conditions, however, many different types of cells produce the molecule at the basal level to maintain tissue equilibrium. In the current study, researchers demonstrated that during cancer progression of breast cells, nitric oxide levels in fact plummet due to the reduction of the functionality of the enzyme that produces nitric oxide. To confirm how that process works out in a live animal, the team artificially blocked the mammary glands of healthy developing mice from producing nitric oxide. This suppression led to multiple precancerous lesions because of an upswing in tumorinducing proteins, independently of causing mutations. In contrast, the researchers introduced sepiapterin into precancerous cells. Sepiapterin is a naturally occurring compound that facilitates the function of nitric oxide-producing enzymes. After treatment, nitric oxide levels normalized, the expression of tumorinducing proteins decreased, and precancerous cells started acting like normal cells. This finding strongly suggests that sepiapterin could be a powerful treatment for the hundreds of thousands of women diagnosed with early-stage breast cancer each year. The researchers are currently testing whether sepiapterin could also be utilized for breast cancer prevention among people susceptible to developing this disease. Though realizing that potential will require more work. Delivered wholesale, sepiapterin could affect other tissues and organs, which might give cancer an advantage. To eliminate this possibility, the team is currently developing a nanoparticle system for delivering sepiapterin payloads specifically to precancerous lesions in the breast. 